\title{
X-Ray CT Imaging with a Dental Panoramic Apparatus
}

\author{
Koichi Ogawa, Kohei Kawai \\ Graduate School of Science and Engineering, Hosei University \\ 3-7-2 Kajinocho, Koganei, Tokyo 184-8584, Japan \\ ogawa@hosei.ac.jp; kohei.kawai.2x@stu.hosei.ac.jp
}

\section{Extended Abstract}

Dental panoramic radiography is a technique that images teeth and jaw bones aligned on a predefined curved plane [1]. If tomosynthesis technique is used, we can reconstruct an object on any curved plane by selecting a shift value of the shiftand-add operation in the tomosynthesis technique [2]. On the other hand, we cannot reconstruct the three dimensional structure of teeth and jaw bones with the dental panoramic x-ray apparatus. The reasons for this are: (1) the typical width of a detector used for the panoramic imaging is as small as less than $1 \mathrm{~cm}$, and (2) the data acquisition orbit of the panoramic $\mathrm{x}$-ray system is not a circle that is used for the clinical x-ray CT system. For these reasons, when a dentist needs an X-ray cone beam CT image of a patient, two different systems (panoramic x-ray system and X-ray CT system) are required, and some manufacturers ship two such data acquisition systems with two different detectors in one apparatus. The purpose of this study is to make an X-ray CT image with a dedicated panoramic X-ray imaging system. In our proposed method, we used a small detector with a size of 50 pixels in width and pixel size of $0.1 \times 0.1 \mathrm{~mm}^{\wedge} 2$. The number of raw data was 3600 , which is a typical number used for a dental panoramic x-ray system [2] that enables tomosynthesis technique. The detector and xray tube rotated circularly, but the center of the rotation moved linearly according to the predefined orbit. We transformed the complicated orbit to a simple circle. Image reconstruction was performed with a maximum likelihood expectation maximization (ML-EM) algorithm [3]. The size of the imaging area was $230 \times 230$ pixels with a pixel size of $1 \times 1 \mathrm{~mm}^{\wedge} 2$. In this imaging we could use only a small detector, thus making necessary a large number of iterations. And so, we used a general purpose graphical processing unit (GP-GPU: NVIDIA Tesla P-100) with CUDA software. The performance of our proposed method was evaluated with simulations using numerical phantoms and images of jaw bone areas with teeth. First, we calculated projection data of each phantom with a detector-source orbit used for the measurement of panoramic x-ray imaging. Then, we reconstructed images of each object with the ML-EM method. We evaluated the quality of images with the root mean square error between the original and reconstructed images. And we increased the number of iterations up to $1,000,000$. The quality of an image was acceptable with the iteration number of 500,000. And it took about 2.43 hours to reconstruct acceptable images. In conclusion, we could successfully reconstruct an X-ray CT image of teeth and jaw bones measured with a panoramic x-ray system.

\section{References}

[1] O. E. Langland, R. P. Langlais, M. D. McDavid, et al., "Panoramic radiology," 2nd ed, Lea \& Febiger, Philadelphia, 1989.

[2] K. Ogawa, R. P. Langlais, M. D. McDavid, et al., "Development of a new dental panoramic radiographic system based on a tomosynthesis method," Dentomaxillofacial Radiology, vol. 39, pp. 47-53, 2010.

[3] K. Lange, M. Bahn, R. Little, "A theoretical study of some maximum likelihood algorithms for emission and transmission tomography," IEEE Trans. Med. Imag., vol. MI-6, pp. 106-114, 1987. 